\title{
Thermally assisted resonant quantum tunneling of magnetization in $\mathrm{Fe}_{8}$ clusters
}

\author{
X. X. Zhang ${ }^{\text {a) }}$ \\ Department of Physics, The Hong Kong University of Science and Technology, Clear Water Bay, Kowloon, \\ Hong Kong, China
}

J. M. Hernandez, E. del Barco, and J. Tejada

Universitat de Barcelona-Xerox Laboratori, Facultat de Fisica, Univ. de Barcelona, 08028 Barcelona, Spain

\author{
A. Roig and E. Molins \\ Institut de Ciencia de Materials de Barcelona, Campus UAB-08193 Bellaterra, Spain \\ K. Wieghardt \\ Max-Planck-Institut fuer Strahlenchemie, Stiftstrasse 34-36, D-45470 Mulheim, Germany
}

\begin{abstract}
Magnetic ac susceptibility of randomly arranged crystallites (powder sample) and well-oriented $\mathrm{Fe}_{8}$ crystallites has been measured as a function of applied magnetic field, temperature, and frequency. The $\mathrm{Fe}_{8}$ clusters, made of eight iron ions and with formula $\left[(\operatorname{tacn})_{6} \mathrm{Fe}_{8} \mathrm{O}_{2}(\mathrm{OH})_{12}\right]^{8+}$, have a ground state $S=10$. From the magnetization data $M(H)$ with the dc field, $H$, applied parallel and perpendicular to the easy axis of the aligned sample and $\chi^{\prime \prime}(T)$ data for different frequencies, the anisotropy field $H_{\text {an }} \sim 5 \mathrm{~T}$ and energy barrier $U / k_{B}=26.9 \mathrm{~K}$ were obtained. Peaks in the curves of $\chi^{\prime}(H)$ were clearly observed at fields $H_{n}=n H_{0}$, with $n=0, \pm 1$ for powder sample where $H_{0}$ $=2.4 \mathrm{kOe}$; and $n=0, \pm 1, \pm 2$, for an oriented sample where $H_{0}=2.2 \mathrm{kOe}$. These peaks appearing at $H_{n}=n H_{0}$ in the curves of $\chi^{\prime}(H)$ can be well described by the thermally assisted resonant quantum tunneling of magnetization. (C) 1999 American Institute of Physics.
\end{abstract}

[S0021-8979(99)75708-7]

Recently, resonant quantum tunneling of magnetization has been found in molecular magnet $\mathrm{Mn}_{12},{ }^{1-5}$ and antiferromagnetic ferritin particles, ${ }^{6}$ which is different from the quantum tunneling of magnetization studied previously. ${ }^{7,8}$ The resonant tunneling of spins (or magnetization) is said to be thermally assisted, that is, the measured magnetization change (or susceptibility) caused by the resonant tunneling effect is governed not only by the tunneling rate of the dominant spin level(s) but also by the thermal population of the very spin level(s) simultaneously. It was found that the tunneling effect can be tuned by temperature and applied magnetic field, which resulted in the observation of some characteristic features [e.g., the jumps equally spaced in magnetization data $M(H)^{1-4}$ and peaks in the real part of susceptibility $\left.\chi^{\prime}(H)\right] .{ }^{5}$ Although the effects of the applied dc field on tunneling have also been observed in magnetic nanoparticles, no fine features have been observed. ${ }^{7}$ In the studies of magnetic nanoparticles, ${ }^{7}$ except the antiferromagnetic ferritin particles, there are a large number of spins (a sizable magnetic moment) in each particle involved in a tunneling event. This large number of spins and not very high anisotropy of the particles will normally lead to an almost continuous spectrum of the spin levels. Consequently no fine feature corresponding to the discrete levels will be observed. Resonant tunneling of magnetization has also been a subject of intensive theoretical studies. ${ }^{9,10}$

Tunneling of magnetization in a $\mathrm{Fe}_{8}$ powder sample was

a)Electronic mail: phxxz@ust.hk recently observed by measuring the $M(H)$ at very low temperatures. ${ }^{11}$ It was suggested that a possible source of tunneling in the $\mathrm{Mn}_{12}$ system could be a small transverse magnetic field, perhaps of dipolar or hyperfine origin, ${ }^{9}$ whereas the $\mathrm{Fe}_{8}$ system is described by the spin Hamiltonian with the transverse anisotropy, which may require separate theoretical investigation. ${ }^{10}$ In this paper we report on the magnetization and ac-susceptibility study on the $\mathrm{Fe}_{8}$ clusters to gain a deeper understanding of the physics underlying the resonant tunneling of magnetization.

$\mathrm{Fe}_{8}$ clusters with formula $\left[(\operatorname{tacn})_{6} \mathrm{Fe}_{8} \mathrm{O}_{2}(\mathrm{OH})_{12}\right]^{8+}$, where tacn is the organic ligand trizacyclononane, ${ }^{12}$ have approximate $D_{2}$ symmetry. Magnetic measurements showed that a $\mathrm{Fe}_{8}$ cluster has a spin ground state $S=10$, resulting from the competing antiferromagnetic coupling between eight $\mathrm{Fe}^{3+}$ ions of spin $s=5 / 2 .^{13}$ The materials used in this experiment are pure $\mathrm{Fe}_{8}$ crystallites with an average size of $0.2 \times 0.1 \times 0.1 \mathrm{~mm}$. Since it is impossible to measure only one crystallite on the Quantum Design superconducting quantum interference device (SQUID) magnetometer, a sample composed of aligned crystallites embedded in a nonmagnetic matrix was used. After putting the mixture of $\mathrm{Fe}_{8}$ crystallites and epoxy in a field of $9.6 \mathrm{~T}$ for $12 \mathrm{~h}$, a solid cylinder with a diameter of $3 \mathrm{~mm}$ and length of $6 \mathrm{~mm}$ was obtained. The good alignment of the crystallites is confirmed by the dc-magnetization data shown in Fig. 1.

Magnetization as a function of temperature was measured in zero field cooled and field cooled processes in a small field of 5 Oe from 1.8 to $40 \mathrm{~K}$, no blocking phenomenon was observed, in agreement with a previous article. ${ }^{11}$ 


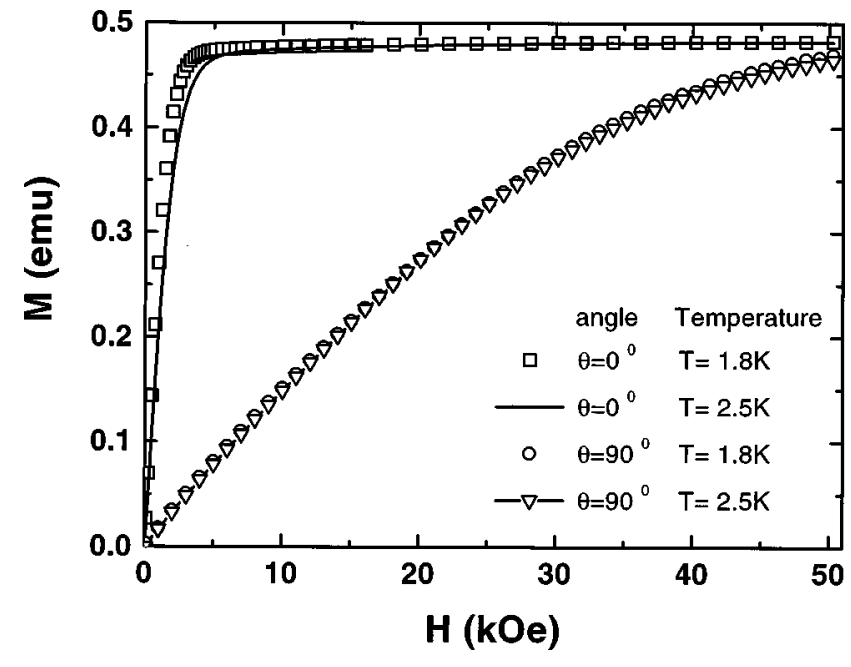

FIG. 1. Magnetization as a function of dc-magnetic field applied parallel and perpendicular to the easy axis of the sample, obtained at 1.8 and $2.5 \mathrm{~K}$.

Figure 1 shows the magnetization as a function of dcmagnetic field, $M(H)$, obtained at 1.8 and $2.5 \mathrm{~K}$, with the field applied parallel and perpendicular to the axis of the cylindrical sample. It is clearly seen that the magnetization curves obtained at 1.8 and $2.5 \mathrm{~K}$ are almost superposed, indicating a very weak temperature-dependent behavior of $M(H)$ near the blocking temperature $1.3 \mathrm{~K} .{ }^{11}$ Therefore, we can consider that the magnetization curves measured at $1.8 \mathrm{~K}$ should possess qualitatively the characteristics of that measured below the blocking temperature. As seen in Fig. 1, the behavior of $M(H)$ for $H$ parallel and perpendicular to the axis of the sample are completely different. For $H$ parallel to the sample axis, $M$ saturated at quite low field (at $\sim 5 \mathrm{kOe}$ ); whereas, when $H$ was applied perpendicularly to the sample axis, $M$ just nearly saturated up to $50 \mathrm{kOe}$. The quality of alignment of the crystallites can be checked by the high field magnetization $M(5 \mathrm{~T})$ and the saturation magnetization $M(0)$ which is obtained by extrapolating the magnetization curve (for $H$ parallel to the axis of the sample) in the range of 5 and $1 \mathrm{~T}$ to $H=0$. In Fig. $1, M(5 \mathrm{~T}) / M(0) \approx 0.99$ suggests a misorientation no more than $1^{\circ}$ and an Ising-type anisotropy. ${ }^{14}$ The anisotropy field can be taken approximately to be $5 \mathrm{~T}^{15}$

Figure 2 shows the real part $\chi^{\prime}(T)$ and imaginary part $\chi^{\prime \prime}(T)$ of the susceptibility versus temperature for $f=250$, 499, 997, and $1490 \mathrm{~Hz}$ obtained on the aligned crystallites. It is evident that peaks in $\chi^{\prime}(T)$ and $\chi^{\prime \prime}(T)$ shift to high temperatures with increasing $f$. For a system composed of identical magnetic clusters, $\chi^{\prime \prime}(T)$ shows a maximum at temperature $T_{p}$ when $2 \pi f \tau=1$. The relaxation time $\tau$ is given by $\tau=\tau_{0} \exp \left(U / k_{B} T\right)$, where $U$ is the anisotropy energy barrier for flipping the magnetic moment of the particle; $\tau_{0}$ is the characteristic time of the system or the reciprocal of attempt frequency $f_{0}$. Therefore, by fitting $T_{p}$ obtained from Fig. 2 for different frequencies to $\ln f=\ln \left(f_{0} / 2 \pi\right)-\left(U / k_{B} T_{p}\right), f_{0}$ $=1.54 \times 10^{8} \mathrm{~Hz}$, and $U / k_{B}=26.94 \mathrm{~K}$ were obtained. The energy barrier $U / k_{B}=26.72 \mathrm{~K}$ and the attempt frequency $f_{0}$ $=1.39 \times 10^{8} \mathrm{~Hz}$ have also been obtained from the

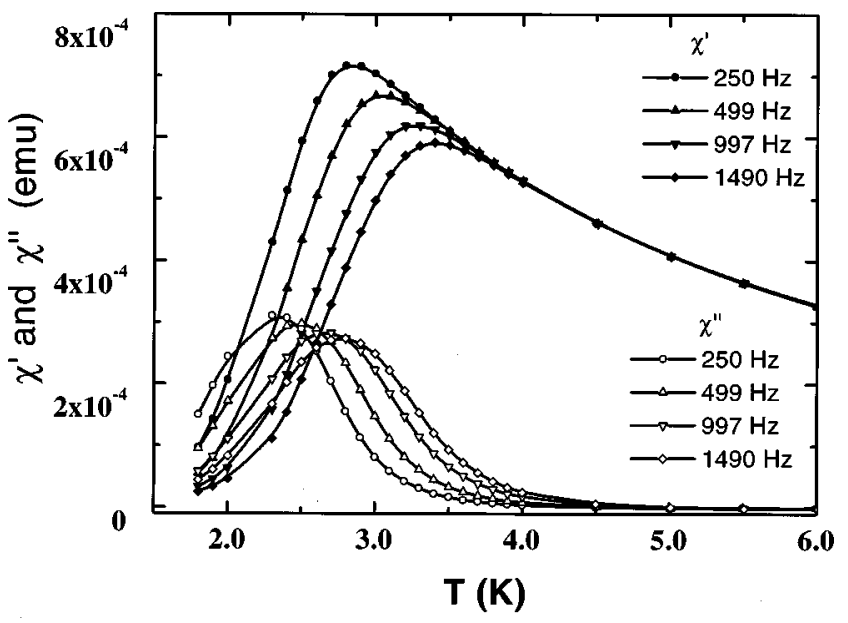

FIG. 2. Temperature-dependent ac-magnetic susceptibility obtained on aligned crystallites in zero dc field with different frequencies.

temperature-dependent susceptibility measured on the powder sample, consistent with the values obtained on the oriented sample.

The ac susceptibility as a function of dc field has been measured at different temperatures $(1.8-4 \mathrm{~K})$ with different frequencies $(1-1500 \mathrm{~Hz})$ for the aligned sample and the powder sample. Figures 3(a) and 3(b) show the fielddependent $\chi^{\prime}(H)$ obtained at $2.5 \mathrm{~K}$ for the powder sample and aligned sample, respectively. It is evident that two peaks

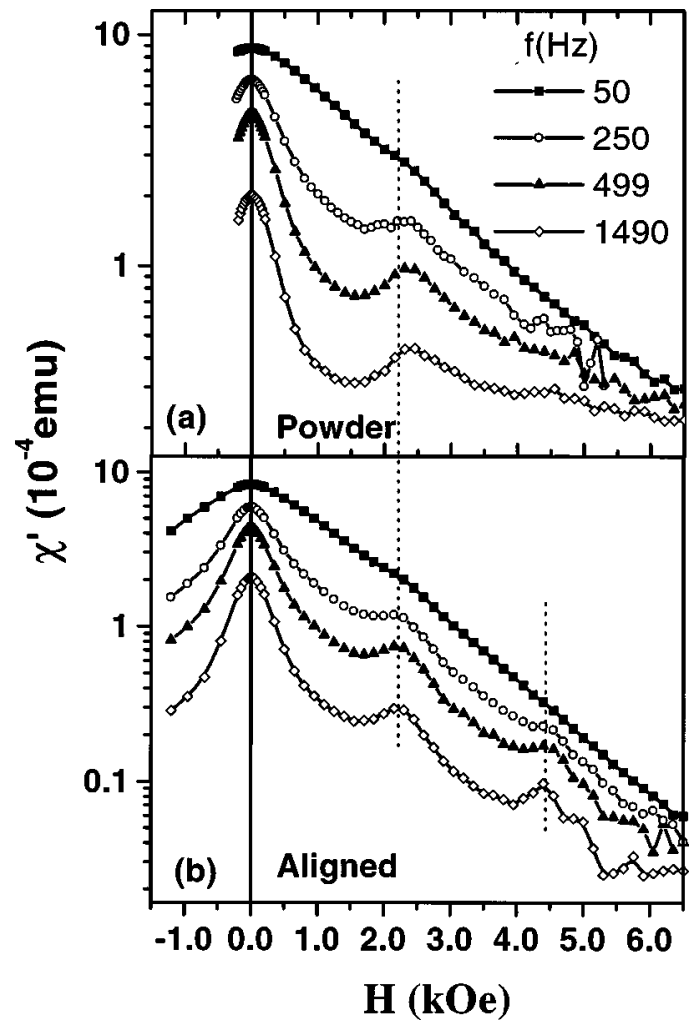

FIG. 3. Real part of ac-magnetic susceptibility as a function of applied dc field obtained on: (a) powder sample (random distributed crystallites) and (b) aligned crystallites. 


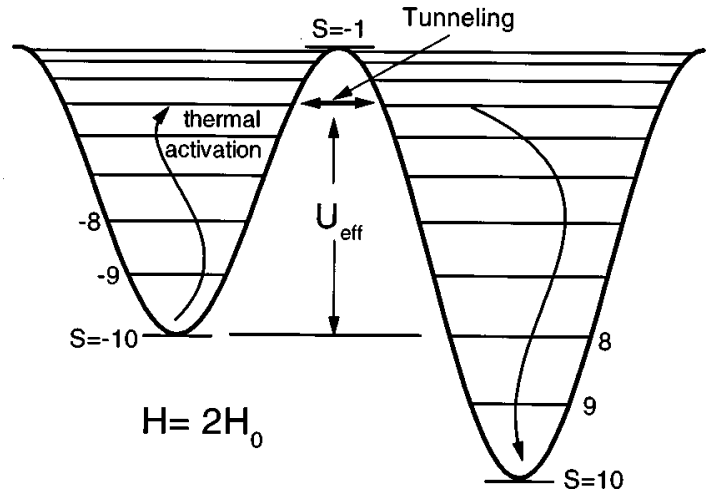

FIG. 4. Schematic diagram of thermally assisted resonant tunneling process. Tunneling occurs when the field brings levels in opposite wells into resonance. The effective energy barrier $U_{\text {eff }}$ determined by the level dominating the resonant tunneling.

at $H=0$ and $\sim 2400$ Oe and three peaks at $H_{n}=n H_{0}$ with $n=0,1,2$ and $H_{0}=2.2 \mathrm{kOe}$ appear in $\chi^{\prime}(H)$ for the random and aligned sample, respectively.

These peaks indicate that the relaxation rate increases markedly at regular intervals of the magnetic field when field is applied parallel to the easy axis of magnetization. The similar phenomenon observed in $\mathrm{Mn}_{12}$ is attributed to resonant tunneling between energy levels in opposite well of the double-well potential. This interpretation is based on a single spin of $S=10$ model with strong uniaxial anisotropy $(\mathcal{H}=$ $-D S z^{2}-g \mu_{B} \mathbf{S} \cdot \mathbf{H}$, where $D$ is the anisotropy energy) for which energy levels corresponding to the spin-up and spindown in the opposite wells coincide at fields $H_{n}$ $=n D / g \mu_{B} B=n H_{0}$. As illustrated in Fig. 4, pairs of levels become degenerate at regular intervals of field, and tunneling occurs between states near the top of barrier. In this process, spins first populate to the high energy levels by thermal activation, then tunnel to the opposite side of the double well through the particular level(s), and decay to the bottom of the well. The particular level which dominates the magnetic transition (susceptibility) determines the effective energy barrier (Fig. 4) involved in the relaxation process. This effective energy barrier oscillates with applied magnetic field along the easy axis. Consequently, the relaxation rate will oscillate with the applied field.

As known that $\mathrm{Fe}_{8}$ clusters have a spin $S=10$ and an Ising-type anisotropy (the anisotropy field $H_{a} \sim 5 \mathrm{~T}$ ) indicated by Fig. 1, we can apply the above model to $\mathrm{Fe}_{8}$ clusters. For the $\mathrm{Fe}_{8}$ cluster, the resonant field $H_{n}=n H_{0}$ with $H_{0}=D / g \mu_{B}=H_{a} / 2 S=2.5 \mathrm{kOe}$ which are in agreement with the $H_{0}$ observed in Fig. 3 and the values obtained in the ac-susceptibility measurements. ${ }^{11}$
By optimizing the parameters which govern the tunneling susceptibility such as temperature, ac-field frequency, and magnetic field, the peak corresponding to $\mathrm{H}_{3}=3 \mathrm{H}_{0}$ $=6.6 \mathrm{kOe}$ is observed at $H=6.56 \mathrm{kOe}$ in $\chi^{\prime}(H)$ measured at $T=2 \mathrm{~K}$ with $f=500 \mathrm{~Hz}$. The only peak (except the peak at $H=0$ ) observed in the powder sample might be caused by the random distribution of easy axis which leads to a broad distribution of the resonant tunneling fields. The broad distribution might smear out the higher order resonant tunneling peaks, say, for $n \geqslant 2$. Another feature that should be noted is that the resonant field $H_{0}=2.4 \mathrm{kOe}$ in the powder sample is higher than that in the aligned sample. This is due to the fact that the position of resonance in $\chi(H)$ only depends on the field applied along the easy axis of magnetization. The field along the easy axis is $H \cos \theta$, where $\theta$ is the angle between the applied field and easy axis, therefore the resonance should always appear at a higher total applied field in a powder sample than in aligned samples. ${ }^{4}$

$\mathrm{We}$, therefore, conclude that the peaks appearing in $\chi^{\prime}(H)$ at fields $H_{n}=n H_{0}$ can be ascribed to thermally assisted resonant tunneling of spins. The anisotropy field of $\mathrm{Fe}_{8}$ clusters has been extracted from the dc-magnetization data, which is agreement with the electron paramagnetic resonance experiment. ${ }^{14}$

X.X.Z. is thankful for support from RGC of Hong Kong Grant Nos. HKUST6111/98P and DAG97/98. SC27).

${ }^{1}$ J. R. Friedman, M. P. Sarachik, J. Tejada, and R. F. Ziolo, Phys. Rev. Lett. 76, 3830 (1996).

${ }^{2}$ J. M. Hernandez, X. X. Zhang, F. Luis, J. Bartolome, J. Tejada, and R. F. Ziolo, Europhys. Lett. 35, 301 (1996).

${ }^{3}$ L. Thomas et al. Nature (London) 383, 145 (1996).

${ }^{4}$ J. M. Hernandez, X. X. Zhang, F. Luis, J. Tejada, J. R. Friedman, M. P. Sarachik, and R. F. Ziolo, Phys. Rev. B 55, 5858 (1997).

${ }^{5}$ F. Luis, J. Bartolome, J. F. Fernandez, J. Tejada, J. M. Hernandez, X. X. Zhang, and R. F. Ziolo, Phys. Rev. B 55, 11448 (1997).

${ }^{6}$ J. Tejada, X. X. Zhang, E. de Barco, J. M. Hernandez, and E. M. Chudniovsky, Phys. Rev. Lett. 79, 1754 (1997); 81, 736 (1998); M. Hanson, J. Johansson, and S. Morup, ibid. 81, 735 (1998).

${ }^{7}$ X. X. Zhang, J. M. Hernandez, J. Tejada, and Ziolo, Phys. Rev. B 54, 4101 (1996); J. Tejada, X. X. Zhang, and E. M. Chnovsky, ibid. 47, 14977 (1993); X. X. Zhang et al., ibid. 53, 3336 (1996).

${ }^{8}$ M. M. Ibrahim, S. Darwish, and M. Seehra, Phys. Rev. B 51, 2955 (1995).

${ }^{9}$ N. V. Prokof'ev and P. C. E. Stamp, Phys. Rev. Lett. 80, 5794 (1998); A. Chiolero and D. Loss, ibid. 80, 169 (1998); A. Fort et al., ibid. 80, 612 (1998); F. Hartmann-Boutron, P. Politi, and J. Villain, Int. J. Mod. Phys. A 10, 2577 (1996).

${ }^{10}$ E. M. Chudnovsky and D. A. Garanin, Phys. Rev. Lett. 79, 4649 (1997); D. A. Garanin and E. M. Chudnovsky, Phys. Rev. B 56, 11102 (1997).

${ }^{11}$ C. Sangregotio et al., Phys. Rev. Lett. 78, 4645 (1997).

${ }^{12} \mathrm{~K}$. Wieghardt, K. Phol, I. Jibril, and G. Huttner, Angew. Chem. Int. Ed. Engl. 23, 77 (1984).

${ }^{13}$ C. Delfs et al., Inorg. Chem. 32, 3099 (1993).

${ }^{14}$ A.-L. Barra et al., Europhys. Lett. 35, 133 (1996).

${ }^{15}$ M. Novak and R. Sessori, NATO ASI Ser., Ser. E 301, 171 (1995). 